\title{
Review
}

\section{Omicron Variant: Secrets Behind the Great Escape Artist}

\author{
Henry Sutanto ${ }^{1}$ and Djoko Santoso ${ }^{2}$ \\ 1 Department of Physiology and Pharmacology, State University of New York (SUNY) Downstate Health \\ Sciences University, Brooklyn, NY \\ 2 Department of Internal Medicine, Faculty of Medicine, Airlangga University, Surabaya, Indonesia \\ * Correspondence: henry1988md@gmail.com
}

\begin{abstract}
The 2019 Coronavirus Disease (COVID-19) is a major cause of morbidity and mortality worldwide. Since late November 2021, Omicron variant has emerged as the primary cause of COVID-19 and caused a huge increase in the reported incidence around the world. To date, 32-34 spike mutations have been reported, 15 of which were located in the receptor-binding domain that interacts with the cell surface of the host cells, while the rest were located in the N-terminal domain and around the Furin cleavage site. At present, both the genomic and clinical profiles of this novel variant are being investigated. Here, we aim to discuss the recent reports on the transmissibility and severity of Omicron variant from both the genetic and clinical perspectives. Afterward, we also take the chance to deliver our personal view on the topic.
\end{abstract}

Keywords: COVID-19; pandemic; SARS-CoV-2; omicron variant; emerging disease; global health; virus; genome; mutation

\section{Introduction}

The 2019 Coronavirus Disease (COVID-19) has been a major cause of morbidity and mortality worldwide since December 2019. To date, the Severe Acute Respiratory Syndrome Coronavirus type-2 (SARS-CoV-2) has infected more than 400 million people and contributed to the death of more than 5.5 million individuals around the world (1). Intriguingly, after more than 2 years of its existence, the infection rate remains high and the pandemic hasn't shown any clear sign of its resolution anytime soon. Following the devastating impacts of the B.1.617.2 (Delta) variant, predominantly in health and socioeconomic sectors, there was a high expectation that the viral disease could be "tamed" by the rapid, collaborative and evolutionary development of COVID-19 vaccines. Indeed, the massive vaccination program in several countries has successfully reduced the fatality of the disease and together with the implementation of a strict Public Health and Social Measures (PHSM), the infection rate could also be lowered (2-5). For example, in the United States, COVID-19 vaccines reduced the overall attack rate (i.e., number of new cases during specified time interval divided by the total population at start of time interval) in the vaccinated individuals by $4.4 \%$ on day 300 from the start of vaccination $(9 \%$ in the unvaccinated group vs. $4.6 \%$ in the vaccinated group), as well as the rate of hospitalization, Intensive Care Unit (ICU) occupancy, incidence of major adverse events and mortality (2). Despite the reported effectivity decline of BNT162b2 and ChAdOx1 vaccines against Delta variant (10-13\% and 16\% lower than B.1.1.7 (Alpha) variant, respectively) (6), vaccination was proven to remain effective in reducing infection and accelerating viral clearance (3), as well as lowering mortality caused by the Delta variant (5).

However, a glimpse of hope to end the pandemic was once again challenged by the presence of the new COVID-19 B.1.1.529 (a.k.a. Omicron) variant. Since its first reported appearance in South Africa in November 2021, it has rapidly taken the attention of experts around the world. A few days after its appearance, the World Health Organization immediately classified Omicron as a Variant of Concern (VoC). Since then, it has vastly spread from Africa to Europe, then Asia, Australia and America. Since early January 2022, Omicron variant has become the major COVID-19 variant in most countries (7) and 
contributed to the rise of COVID-19 incidence from $\sim 600,000$ new cases in late November 2021 to 3.5 million cases in late January 2022 (1).

\section{The genomic basis of Omicron variant}

A study conducted based upon the genome sequencing data of 108 samples collected from patients infected with Omicron variant (8) revealed that this variant possessed 61-64 mutations, 54 of which were Single Nucleotide Polymorphisms (SNPs). Of those, $34 \mathrm{mu}-$ tations were positioned at the spike (S) proteins and 32 of those spike mutations were nonsynonymous. This number was significantly higher than that of its predecessors (8). Importantly, the mutations in Omicron variant were reported to be present in all three key regions in the spike of SARS-CoV-2: The Receptor Binding Domain (RBD), N-terminal Domain (NTD) and Furin Cleavage Site (FCS). Fifteen of the spike mutations were located in the RBD, a region that is responsible for the viral attachment to the cell surface (Table 1).

Table 1. Reported mutations in S-proteins of Omicron variant.

\begin{tabular}{|c|c|c|}
\hline NTD & RBD & FCS \& adjacent \\
\hline A67V a,b,\# & G339D a,b,\# & T547K a,b,\# \\
\hline IHV68I a & S371L a,b & D614G a,b \\
\hline $\operatorname{del} 69-70 \mathrm{b, \#}$ & S373P a,b & H655Y a,b,\# \\
\hline T95I a,b & S375F a,b & N679K a,b,\# \\
\hline GVYY142D a & $\mathrm{K} 417 \mathrm{~N}$ a,b & P681H a,b,\# \\
\hline del142-144 b & N440K a,b & N764K a,b \\
\hline Y145D b & G446S a,b & D796Y a,b,\# \\
\hline $\mathrm{NL}^{211 \mathrm{I}^{\mathrm{a}}}$ & $\mathrm{S} 477 \mathrm{~N} \mathrm{a,b}$ & N856K a,b,\# \\
\hline del211 b & $\mathrm{T} 478 \mathrm{~K} \mathbf{a}, \mathbf{b}$ & Q954H a,b,\# \\
\hline $\mathrm{L}^{212 \mathrm{I}^{\mathrm{b}}}$ & $\mathrm{E} 484 \mathrm{~A}^{\mathrm{a}, \mathrm{b}}$ & N969K a,b,\# \\
\hline \multirow[t]{5}{*}{ Ins214EPE b } & Q493R a,b & L981F a,b,\# \\
\hline & G496S a,b & \\
\hline & Q498R a,b & \\
\hline & N501Y a,b & \\
\hline & $\mathrm{Y} 505 \mathrm{H}$ a,b & \\
\hline
\end{tabular}

${ }^{\mathrm{a}}$ was reported by Ma et al. (8) and ${ }^{\mathrm{b}}$ was reported by the United States Centers for Disease Control and Prevention [CDC] (9). The mutations that are potentially suitable for Omicron screening are marked by (\#). This was assessed based on the uniqueness of these mutations for Omicron variant according to the Communicable Diseases Genomics Network (CDGN; https://www.cdgn.org.au/) per 20 December 2021.

As displayed in Table 1, the N501Y mutation that converts amino acid asparagine to tyrosine at position 501 was identified in Omicron variant. This particular SNP enhanced the binding affinity of the RBD to Angiotensin Converting Enzyme type-2 (ACE2) receptor in the surface of the host cell (10). As a consequence, Omicron variant could have a stronger attachment to the host cells than some of the other COVID-19 variants, fostering its transmissibility. Meanwhile, the mutation Q498R (converting glutamine to arginine at position 498) of the RBD alone negatively affected the protein stability and binding. However, due to its known epistatic effect with N501Y, the combination of both mutations was shown to increase the affinity of the RBD to ACE2 receptor by 4-fold (11). In addition to their effect on the binding affinity to ACE2 receptor, Omicron-associated mutations in the RBD could also promote the escape from existing neutralizing antibodies. In the study done by Cao et al. (12), the effect of Omicron-associated mutations in RBD region on neutralizing antibodies was assessed using a high throughput yeast display screening. As the result, K417N, G446S, E484A and Q493R mutations assisted the virus to evade neutralizing antibodies, especially the ones which epitope overlapped with the ACE2 binding motif (epitope group A-D). Also, there was evidence that G339D, S371L and N440K 
mutations of the RBD could facilitate the virus evasion from other neutralizing antibodies (epitope group E-F). Overall, of the 247 antibodies tested, 85\% were evaded by Omicron, suggesting the potentially low efficacy of preexisting neutralizing antibodies against Omicron variant (12).

Several mutations in the NTD region were shown to have significant effect on viral infectivity and the modulation of immune evasion. For example, the del69-70 boosted the infectivity of Alpha variant through the elevation of cleaved spike incorporation into virions (13). Conversely, T95I mutation that was previously identified in B.1.617.1 variant seems not to be substantially involved in immune evasion due to its location outside of the antigenic supersite (14). Interestingly, an incidence of vaccine breakthrough infection was reported in COVID-19 patients harboring T95I mutation of the NTD, as well as the del142-144 mutation of the NTD and D614G mutation of the FCS, indicating the possible contribution of those mutations on viral immune escape (15). Meanwhile, an evidence of a marked (4-16 fold) reduction of neutralizing capacity of COVID-19 convalescent sera against a recombinant vesicular stomatitis virus carrying SARS-CoV-2 spike protein with Y145D mutation was also reported (16).

The FCS region has been shown to be a key part of SARS-CoV-2 pathogenesis and severity. For instance, a mutant lacking of FCS ( $\triangle$ PRRA) displayed a reduced replication rate in a human respiratory cell line (17). Meanwhile, in regard to Omicron-associated mutations, the H655Y and N679K mutations which are located proximal to the FCS, and the $\mathrm{P} 681 \mathrm{H}$ mutation of the FCS could enhance the SARS-CoV-2 spike cleavage and increase the transmissibility of Omicron variant (9). Moreover, the P681H mutation, which was previously identified in the B.1.1.7 (Alpha) variant, was demonstrated to facilitate the viral resistance against innate immunity (i.e., interferon- $\beta$ ) in lung epithelial cells (18). Yet, another study on this particular mutation did not find any notable change on the spike cleavage, viral entry or intercellular spreading (19). Next, the D614G mutation increased viral replication in human lung epithelia and respiratory tract by fostering the virion stability and infectivity. Moreover, it increased the viral load in the upper respiratory tract, thereby promoting viral transmission (20). However, it is important to note that the effect of such mutations on Omicron pathogenicity may differ from what was reported in previous COVID-19 variants due to distinct interactions among variant-associated mutations.

Next, the change of viral tropism in the Omicron variant is also important to scrutinize. A recent UK-based study showed that Omicron variant was strongly associated with symptoms from upper respiratory tract more than the lower respiratory tract (21). This could be explained by the presence of two Omicron-specific mutations: N764K and N856K. Those mutations were shown to produce cleavage sites for Subtilisin-Kexin Isozyme-1 / Site-1 Protease (SKI-1/S1P) serine protease predominantly situated in the upper airway. Such cleavage sites are important to cleave viral envelope glycoproteins, which modulate SARS-CoV-2 replication and pathogenesis (22). Indeed, Omicron variant was shown to replicate faster in the bronchus than in the lung parenchyma (23). Another study also highlighted that despite the similar viral replication in human nasal epithelial cultures, Omicron variant demonstrated lower replication in lower respiratory and pulmonary cells than the Delta variant (24). Additionally, Omicron spike protein has a lower S1/S2 cleavage efficiency than Delta variant and Omicron tends to avoid cells expressing a high level of transmembrane protease, serine 2 (TMPRSS2). This was due to Omicron's failure in exploiting the TMPRSS2 that promotes cell entry via plasma membrane fusion. As a consequence, the cell entry of Omicron variant was largely mediated through the endocytic pathway $(24,25)$. The fact that SKI-1/S1P was also present in pulmonary macrophages (22) could suggest the potential consequences of N764K and N856K mutations on the host innate immunity. Nevertheless, more studies are needed to confirm this notion.

\section{What can we tell from the $\mathrm{Ct}$ value of patients with Omicron variant?}


Quantitative Polymerase Chain Reaction (qPCR) is currently used as a gold standard of diagnostics for COVID-19. Briefly, it works by amplifying or copying small amount of the genetic materials (e.g., Ribonucleic Acid / RNA) isolated from the upper respiratory tract specimen of the suspected / infected individuals. Using a technique called reverse transcription and an enzyme namely reverse transcriptase, a single-stranded RNA is converted into a double-stranded deoxyribonucleic acid (DNA), which subsequently undergoes a separation process. Using a primer attached to each of this strand, two copies of identical DNA can be generated. In qPCR, fluorescent dyes are also attached to each successful duplication, so that the amount can be quantified. Finally, the amplification cycle is repeated for 20-40 times to obtain hundreds of copies of the viral genetic materials.

Cycle threshold $(\mathrm{Ct})$ value is the number of cycle required for the fluorescent signal to reach the given threshold, meaning that a high $\mathrm{Ct}$ value indicates a smaller viral load. Conversely, the lower the $\mathrm{Ct}$ level the greater the amount of identified target RNA in the sample. A recent study assessing the real-time (RT) qPCR data from 10,324 specimens and comparing 97 Omicron against 107 Delta variants infections reported that the Ct values was higher for Omicron infections than for Delta infections (Omicron: Ct 23.3 vs. Delta: Ct 20.5), suggesting a lower peak viral RNA of the Omicron variant. Moreover, the clearance phase for Omicron was shorter while the clearance rate was similar with Delta variant (26). Similarly, a study conducted in France also reported a significantly higher $\mathrm{Ct}$ value for Omicron infection than the non-Omicron variants (27). These findings suggest that the high transmissibility of Omicron variant might not be due to a high viral load in the upper respiratory tract, despite the presence of D614G mutation which was known to increase viral replication in the upper respiratory tissue (20).

\section{Clinical features of Omicron variant}

A recent observational study conducted in Texas, the United States, from late December 2021 to early January 2022 reported that Omicron variant displayed some different clinical patterns than its predecessors (28). Compared to patients infected by Alpha and Delta variants, these patients were younger and predominantly female. The number of patients requiring hospitalization was significantly lower in Omicron than in Alpha and Delta variants infection ( $19.8 \%$ vs. $54.6 \%$ and $43.1 \%$, respectively). Of those who were hospitalized, the average length of stay was significantly shorter in Omicron than Alpha and Delta (3.2 days vs. 5.1 days and 5.4 days, respectively). Moderate to severe cases (e.g., number of patients requiring Extracorporeal Membrane Oxygenation [ECMO], mechanical ventilation and high-flow oxygenation) and mortality were also lower in Omicron infection. As expected, while Alpha and Delta variants affected mostly the unvaccinated individuals, Omicron variant proportionally infected both the unvaccinated (44.1\%) and vaccinated people (55.9\%) (28). These findings and comparable results from another study (29) confirm the hypothesis that Omicron variant causes less severe disease, resulted in a lower hospitalization rate. Importantly, the fact that Omicron caused an increased vaccine breakthrough incidence (28) needs to be swiftly responded by speeding up the vaccination booster campaign. The third vaccine (booster) dose has been shown to rescue and broaden the viral neutralization (30-32). Therefore, it is expected to be beneficial to tackle this emerging COVID-19 variant.

It is also crucial to acknowledge that although studies have shown compelling evidence of a less severe disease and a lower hospitalization due to Omicron infection, the high transmissibility of this variant might still impact the healthcare system readiness and increase the absolute number of hospitalization (33). These conditions would cause a severe delay on the non-COVID-19 patient care and might result in bigger indirect consequences than we expected. Also, the effect of Omicron variant in immunocompromised individuals, and patients with concomitant diseases and comorbidities is still unknown. In general, these individuals have an impaired immune response against infections, a condition that favors Omicron and other COVID-19 variants. Nonetheless, the effect of Omicron on this special population is yet to be explored. 


\section{Summary}

Overall, the known genomic profile of Omicron variant hinted a high affinity to ACE2 receptor, a high transmissibility and a likelihood to evade neutralizing antibodies (24), either from the previous vaccination $(30,34)$ or prior infection(s) (35). The fact that Omicron variant had a lower predisposition to damage lower respiratory tract and pulmonary tissue could indicate its potentially lower severity than its predecessors (e.g., Delta variant). Indeed, the less severe feature of Omicron has been seen in several observational studies. Nonetheless, the consequences of this variant on special populations (e.g., individuals with altered immune response) remain to be elucidated.

Regardless, implementing an effective PHSM could still be beneficial to limit personto-person transmission of Omicron variant. The application of well-fitting mask, imposing strict physical distancing, cough etiquette and hand hygiene, as well as avoiding crowd remain vital. The World Health Organization has already advised to enhance surveillance with rapid testing, cluster investigations, contact tracing and quarantine, as well as case isolation to cut the chain of transmission (36). Only by a collaborative effort, the COVID19 pandemic could be brought to an end.

\section{References}

1. Worldometer. COVID-19 Coronavirus Pandemic 2022 [Available from: https://www.worldometers.info/coronavirus/.

2. Moghadas SM, Vilches TN, Zhang K, Wells CR, Shoukat A, Singer BH, et al. The Impact of Vaccination on Coronavirus Disease 2019 (COVID-19) Outbreaks in the United States. Clin Infect Dis. 2021;73(12):2257-64.

3. Singanayagam A, Hakki S, Dunning J, Madon KJ, Crone MA, Koycheva A, et al. Community transmission and viral load kinetics of the SARS-CoV-2 delta (B.1.617.2) variant in vaccinated and unvaccinated individuals in the UK: a prospective, longitudinal, cohort study. Lancet Infect Dis. 2022;22(2):183-95.

4. $\quad \mathrm{Ng}$ OT, Koh V, Chiew CJ, Marimuthu K, Thevasagayam NM, Mak TM, et al. Impact of Delta Variant and Vaccination on SARS-CoV-2 Secondary Attack Rate Among Household Close Contacts. Lancet Reg Health West Pac. 2021;17:100299.

5. Sheikh A, Robertson C, Taylor B. BNT162b2 and ChAdOx1 nCoV-19 Vaccine Effectiveness against Death from the Delta Variant. N Engl J Med. 2021;385(23):2195-7.

6. Pouwels KB, Pritchard E, Matthews PC, Stoesser N, Eyre DW, Vihta KD, et al. Effect of Delta variant on viral burden and vaccine effectiveness against new SARS-CoV-2 infections in the UK. Nat Med. 2021;27(12):2127-35.

7. GISAID. Country Submission Count 2022 [Available from: https://www.gisaid.org/hcov19-variants/.

8. Ma W, Yang J, Fu H, Su C, Yu C, Wang Q, et al. Genomic perspectives on the emerging SARS-CoV-2 omicron variant. Genomics Proteomics Bioinformatics. 2022.

9. CDC. Science Brief: Omicron (B.1.1.529) Variant 2022 [Available from: https://www.cdc.gov/coronavirus/2019ncov/science/science-briefs/scientific-brief-omicron-variant.html.

10. Liu Y, Liu J, Plante KS, Plante JA, Xie X, Zhang X, et al. The N501Y spike substitution enhances SARS-CoV-2 infection and transmission. Nature. 2022;602(7896):294-9.

11. Zahradnik J, Marciano S, Shemesh M, Zoler E, Harari D, Chiaravalli J, et al. SARS-CoV-2 variant prediction and antiviral drug design are enabled by RBD in vitro evolution. Nat Microbiol. 2021;6(9):1188-98.

12. Cao Y, Wang J, Jian F, Xiao T, Song W, Yisimayi A, et al. Omicron escapes the majority of existing SARS-CoV-2 neutralizing antibodies. Nature. 2021.

13. Meng B, Kemp SA, Papa G, Datir R, Ferreira I, Marelli S, et al. Recurrent emergence of SARS-CoV-2 spike deletion H69/V70 and its role in the Alpha variant B.1.1.7. Cell Rep. 2021;35(13):109292.

14. McCallum M, Walls AC, Sprouse KR, Bowen JE, Rosen LE, Dang HV, et al. Molecular basis of immune evasion by the Delta and Kappa SARS-CoV-2 variants. Science. 2021;374(6575):1621-6.

15. Hacisuleyman E, Hale C, Saito Y, Blachere NE, Bergh M, Conlon EG, et al. Vaccine Breakthrough Infections with SARSCoV-2 Variants. N Engl J Med. 2021;384(23):2212-8. 
16. Haslwanter D, Dieterle ME, Wec AZ, O'Brien CM, Sakharkar M, Florez C, et al. A Combination of Receptor-Binding Domain and N-Terminal Domain Neutralizing Antibodies Limits the Generation of SARS-CoV-2 Spike Neutralization-Escape Mutants. mBio. 2021;12(5):e0247321.

17. Johnson BA, Xie X, Bailey AL, Kalveram B, Lokugamage KG, Muruato A, et al. Loss of furin cleavage site attenuates SARSCoV-2 pathogenesis. Nature. 2021;591(7849):293-9.

18. Lista MJ, Winstone H, Wilson HD, Dyer A, Pickering S, Galao RP, et al. The P681H mutation in the Spike glycoprotein confers Type I interferon resistance in the SARS-CoV-2 alpha (B.1.1.7) variant. bioRxiv. 2021:2021.11.09.467693.

19. Lubinski B, Fernandes MHV, Frazier L, Tang T, Daniel S, Diel DG, et al. Functional evaluation of the P681H mutation on the proteolytic activation of the SARS-CoV-2 variant B.1.1.7 (Alpha) spike. iScience. 2022;25(1):103589.

20. Plante JA, Liu Y, Liu J, Xia H, Johnson BA, Lokugamage KG, et al. Spike mutation D614G alters SARS-CoV-2 fitness. Nature. 2021;592(7852):116-21.

21. Vihta K-D, Pouwels KB, Peto TE, Pritchard E, House T, Studley R, et al. Omicron-associated changes in SARS-CoV-2 symptoms in the United Kingdom. medRxiv. 2022:2022.01.18.22269082.

Maaroufi H. The N764K and N856K mutations in SARS-CoV-2 Omicron S protein generate potential cleavage sites for SKI1/S1P protease. bioRxiv. 2022:2022.01.21.477298.

Hui KPY, Ho JCW, Cheung MC, Ng KC, Ching RHH, Lai KL, et al. SARS-CoV-2 Omicron variant replication in human bronchus and lung ex vivo. Nature. 2022.

24. Meng B, Abdullahi A, Ferreira I, Goonawardane N, Saito A, Kimura I, et al. Altered TMPRSS2 usage by SARS-CoV-2 Omicron impacts tropism and fusogenicity. Nature. 2022.

25. Zhao H, Lu L, Peng Z, Chen LL, Meng X, Zhang C, et al. SARS-CoV-2 Omicron variant shows less efficient replication and fusion activity when compared with Delta variant in TMPRSS2-expressed cells. Emerg Microbes Infect. 2022;11(1):277-83.

26. Hay JA, Kissler SM, Fauver JR, Mack C, Tai CG, Samant RM, et al. Viral dynamics and duration of PCR positivity of the SARS-CoV-2 Omicron variant. medRxiv. 2022:2022.01.13.22269257.

27. Sofonea MT, Roquebert B, Foulongne V, Verdurme L, Trombert-Paolantoni S, Roussel M, et al. From Delta to Omicron: analysing the SARS-CoV-2 epidemic in France using variant-specific screening tests (September 1 to December 18, 2021). medRxiv. 2022:2021.12.31.21268583.

28. Christensen PA, Olsen RJ, Long SW, Snehal R, Davis JJ, Saavedra MO, et al. Signals of Significantly Increased Vaccine Breakthrough, Decreased Hospitalization Rates, and Less Severe Disease in Patients with Coronavirus Disease 2019 Caused by the Omicron Variant of Severe Acute Respiratory Syndrome Coronavirus 2 in Houston, Texas. Am J Pathol. 2022.

Maslo C, Friedland R, Toubkin M, Laubscher A, Akaloo T, Kama B. Characteristics and Outcomes of Hospitalized Patients in South Africa During the COVID-19 Omicron Wave Compared With Previous Waves. JAMA. 2022;327(6):583-4.

Accorsi EK, Britton A, Fleming-Dutra KE, Smith ZR, Shang N, Derado G, et al. Association Between 3 Doses of mRNA COVID-19 Vaccine and Symptomatic Infection Caused by the SARS-CoV-2 Omicron and Delta Variants. JAMA. 2022.

Perez-Then E, Lucas C, Monteiro VS, Miric M, Brache V, Cochon L, et al. Neutralizing antibodies against the SARS-CoV-2 Delta and Omicron variants following heterologous CoronaVac plus BNT162b2 booster vaccination. Nat Med. 2022.

Schmidt F, Muecksch F, Weisblum Y, Da Silva J, Bednarski E, Cho A, et al. Plasma Neutralization of the SARS-CoV-2 Omicron Variant. N Engl J Med. 2022;386(6):599-601.

Ulloa AC, Buchan SA, Daneman N, Brown KA. Early estimates of SARS-CoV-2 Omicron variant severity based on a matched cohort study, Ontario, Canada. medRxiv. 2022:2021.12.24.21268382.

Collie S, Champion J, Moultrie H, Bekker LG, Gray G. Effectiveness of BNT162b2 Vaccine against Omicron Variant in South Africa. N Engl J Med. 2022;386(5):494-6.

35. Altarawneh HN, Chemaitelly H, Hasan MR, Ayoub HH, Qassim S, AlMukdad S, et al. Protection against the Omicron Variant from Previous SARS-CoV-2 Infection. N Engl J Med. 2022. 
36. WHO. Enhancing response to Omicron SARS-CoV-2 variant 2022 [Available from: https://www.who.int/publications/m/item/enhancing-readiness-for-omicron-(b.1.1.529)-technical-brief-and-priorityactions-for-member-states. 